\title{
The Contribution of Salivary Amylase to Glucose Polymer Hydrolysis in Premature Infants
}

\author{
ROBERT D. MURRAY, BENNY KERZNER, HOWARD R. SLOAN, H. JUHLING MCCLUNG, \\ MERRY GILBERT, AND ANTON AILABOUNI
}

Children's Hospital Research Foundation, Columbus, Ohio 43205

\begin{abstract}
To determine whether salivary amylase of premature infants can function as a surrogate for pancreatic amylase, we evaluated its production in the infant, acid resistance, and hydrolytic potency in a simulated oropharyngeal, gastric, and intestinal environment. The activity of salivary amylase in 11 prematures varied between 1 and $33 \mathrm{U} / \mathrm{ml}$; the isozymic profile and acid resistance of the premature salivary amylase were identical to those of the enzyme of adults. A "modular" formula containing $7 \mathrm{~g} / \mathrm{dl}$ of a ${ }^{14} \mathrm{C}$ labeled long chain glucose polymer with degrees of polymerization ranging between 18 and 29 glucose units was prepared. Salivary amylase, $1.1 \mathrm{U} / \mathrm{ml}$, was added to this formula. The progressive breakdown of the ${ }^{14} \mathrm{C}$ polymers as the milk was subjected to oropharyngeal, gastric, and intestinal phase environments was evaluated by quantifying the liberation of short-chain oligomers from the ${ }^{14} \mathrm{C}$ labeled substrates. The gastric $\mathrm{pH}$ was varied between 2 and 5 and the gastric incubation time was either 5 or 180 min. Substantial gastric phase breakdown only occurred after $3 \mathrm{~h}$ of exposure at the higher pHs of $4(12 \%)$ and 5 $(32 \%)$. During the intestinal phase, salivary amylase activity resumed. Prior gastric phase $\mathrm{pH}$ affected ultimate intestinal phase breakdown, $p<0.001$; after 5 -min gastric phases at pHs ranging from 2 to 5 , the intestinal phase breakdown ranged from 17 to $55 \%$. We conclude that the limited salivary amylase in the saliva of premature infants can produce significant glucose polymer digestion in both the stomach and small intestine but the digestion falls substantially short of that accomplished by usual concentrations of pancreatic amylase. (Pediatr Res 20: 186-191, 1986)
\end{abstract}

Abbreviations

SA, salivary amylase

GP, glucose polymer

$D P$, degree of polymerization

Pancreatic amylase activity is the most effective enzyme for starch and GP hydrolysis. In the first 6 months of life, however, pancreatic amylase is essentially absent; yet newborns, even those prematurely born, assimilate substantial amounts of GPs. This observation has been attributed to the hydrolytic activity of four potential substitutes for pancreatic amylase: SA, glucoamylase, breast milk amylase, and the colonic bacterial flora (1). SA

Received June 3, 1985: accepted September 23, 1985

Correspondence and reprint requests Benny Kerzner, M.B.B.Ch., F.C.P.(SA) Division of Gastroenterology, Children's Hospital, 700 Children's Drive, Columbus, $\mathrm{OH} 43205$.

This study was supported by Grants 1 RO1 HD17506 and 7 RO1 HD18188 from the National Institutes of Health, Grant 74-371 from Ross Laboratories, and Grant 74-228 from the Children's Hospital Research Foundation. activity appears early in gestation and gradually increases with advancing gestational age (2). The enzyme activity in early life is low, however, and the contribution of SA activity to GP digestion during a feeding is not known.

The experiments described herein were undertaken to determine the potential contribution of SA activity to GP hydrolysis. In vitro studies allowed us to analyze the ability of SA to hydrolyze GPs, independent of the contribution of mucosal enzymes or other hydrolytic mechanisms present in the intact organism. To reproduce the conditions in the newborn's intestine in which only minimal salivary amylase activity is available, we employed units of enzymatic activity based on those estimated to be analogous to those present in premature infants. To measure the hydrolysis of the GP in a meal as it progresses through simulated oropharyngeal, gastric, and intestinal phases of digestion, we prepared a uniformly labeled ${ }^{14} \mathrm{C}$-GP substrate representative of the long-chain material in corn starch hydrolysates frequently employed in infant formulae and developed a technique for measuring the progressive liberation of glucose oligomers from this compound.

\section{MATERIALS AND METHODS}

The following materials were obtained from commercial sources: ${ }^{14} \mathrm{C}$ tobacco starch (Pathfinder Laboratories, Inc., St. Louis, MO); a heat-stable bacterial $\alpha$-amylase, Takatherm L-170 (Miles Laboratories, Elkhart, IN); human salivary amylase (Sigma Chemical Company, St. Louis, MO); a commercial partial corn starch hydrolysate, Polycose and a carbohydrate-free soy formula, RCF (Ross Laboratories, Columbus, OH); Bio-Gels P-2 and P-10, 200-400 mesh (Bio-Rad Laboratories, Richmond, CA); Ringer's solution, a commercial electrolyte solution containing (in meq/l): Na-147.5, K-4, Ca-4.5, and Cl-156 (Travenol, Deerfield, IL); XAR-5 autoradiographic film (Kodak, Rochester, NY); LK-5 thin-layer plates (Whatman, Clifton, NJ); Scintiverse Bio-HP scintillation counting fluid (Fisher Scientific Products, Fairlawn, NJ); and Phadebas tablets (Pharmacia, Piscataway, $\mathrm{NJ}$ ); special purpose electrophoresis film (Corning, Palo Alto, $\mathrm{CA}$ ); and $0.45 \mu$ membrane filters (Millipore, Bedford, MA). All chemicals were of reagent grade, and the solvents were not redistilled prior to use.

Preparative Studies for GP Hydrolysis Experiments. Two preliminary studies were required prior to the studies on GP degradation by SA: first, production of a ${ }^{14} \mathrm{C}$-GP containing only longchain elements; and second, estimation of the amount of salivary amylase activity produced per swallow of formula in premature infants.

Preparation and validation of the ${ }^{14} \mathrm{C}$ glucose polymer substrate. Uniformly labeled ${ }^{14} \mathrm{C}$-tobacco starch was partially digested with Takatherm, a bacterial $\alpha$-amylase with endoamylase activity which produces a spectrum of GPs similar to that found in commercial partial corn starch hydrolysates. Two hundred fifty microcuries of ${ }^{14} \mathrm{C}$ tobacco starch $(1.5 \mathrm{mCi} / \mathrm{mg})$ in $2 \mathrm{ml}$ of 
ethanol:water (1:1) was added to an equal volume of a $0.0043 \mathrm{M}$ $\mathrm{CaCl}_{2}$ solution and brought to $58^{\circ} \mathrm{C}$ in a waterbath. Takatherm, diluted 1000 -fold in the same $\mathrm{CaCl}_{2}$ solution, was then added. After $20 \mathrm{~s}$, digestion was stopped by the addition of $0.2 \mathrm{ml}$ of 1.0 $\mathrm{M} \mathrm{HCl}$. The product was lyophilized, reconstituted in $1 \mathrm{ml}$ of sterile water, and applied to a $42 \mathrm{ml}$ Bio-Gel P-10 $(107 \times 0.7$ $\mathrm{cm}$ ) column. Starting $1 \mathrm{ml}$ after the void volume, $7 \mathrm{ml}$ of eluant were collected, pooled, and lyophilized. The average degree of polymerization of this material was determined by molecular sieve chromatography on Bio-Gel P-10 columns according to the method of Porath to be 23 (DP $\left.{ }_{A v G} 23\right)(3,4)$. From the nonradioactive partial corn starch hydrolysate, an equivalent subfraction of long chain GPs was obtained by chromatography on a $1000 \mathrm{ml}(260 \times 2.2 \mathrm{~cm})$ Bio-Gel P-2 column (4). The radioactive and nonradioactive products were mixed to yield a specific activity of $1.4 \mu \mathrm{Ci} / \mathrm{g}$ of carbohydrate.

To establish that the nonradioactive and radioactive components of the GP substrate contained equal concentrations of each polymer and that salivary endoamylase action yielded a similar spectrum of breakdown products, we performed the following experiments.

1) $220 \mathrm{mg}$ of the nonradioactive $\mathrm{DP}_{\mathrm{AVG}} 23$ and $0.2 \mu \mathrm{Ci}$ of the radioactive $\mathrm{DP}_{A V G} 23$ were mixed in a test tube and applied to a $38.5 \times 0.7 \mathrm{~cm}$ column of Bio-Gel P-2. The column was eluted with water at $3 \mathrm{ml} / \mathrm{h}$ and $0.25 \mathrm{ml}$ samples were collected. The fractions were evaluated for carbohydrate content by the phenolsulphuric assay and radioactivity by scintillation spectrometry.

2) $220 \mathrm{U}$ of SA were incubated with carbohydrate-free formula (350 $\mu$ l) containing $20 \mathrm{mg}$ of nonradioactive and $0.2 \mu \mathrm{Ci}$ of radioactive $\mathrm{DP}_{\wedge \mathrm{VG}_{\mathrm{G}}} 23$ at $37^{\circ} \mathrm{C}$ for $30 \mathrm{~min}$. The reaction was stopped with $35 \mu \mathrm{l}$ of $20 \mathrm{~g} / \mathrm{dl}$ trichloroacetic acid and centrifuged at $15,000 \mathrm{rpm}$ for $10 \mathrm{~min}$. A $0.5-\mathrm{ml}$ sample of supernatant was placed on a Bio-Gel P-2 $(38.5 \times 0.7 \mathrm{~cm})$ column and developed with water at $3 \mathrm{ml} / \mathrm{h}$. Following the void volume, $0.75 \mathrm{ml}$ aliquots were collected and analyzed. The carbohydrate content of each test tube, determined by the phenol-sulphuric acid method, and the radioactivity, measured by scintillation spectrometry were expressed as a percentage of the sample applied to the column and compared.

Determination of $S A$ activity for in vitro studies. To estimate the SA activity to be mixed with premature infant formula in an in vitro experiment, one needs to know: the rate of saliva production, the time (per swallow) during which SA is secreted, and the SA activity per $\mathrm{ml}$ of saliva. Both the saliva production rate and the maximum duration of a swallow are known and are 0.15 $\mathrm{ml} / \mathrm{min}$ and $15 \mathrm{~s}$, respectively $(5-7)$. We determined, in separate experiments, the SA activity in the saliva of 13 premature infants with corrected gestational ages (gestational age plus postnatal age) of 24 to $33 \mathrm{wk}$. A size 8 French polyvinyl feeding tube was used to aspirate gently $1.0 \mathrm{ml}$ of saliva from the infant's cheeks. The collection of saliva from the infants was approved by the Columbus Children's Hospital Human Subjects Research Committee. SA activity per $\mathrm{ml}$ of saliva was measured using the Phadebas test. The highest and lowest SA production rates per swallow in prematures were then calculated for use in the GP hydrolysis experiments ( 1.1 and $0.011 \mathrm{U} / \mathrm{ml}$, respectively).

GP Hydrolysis Experiments Utilizing Four Salivary Amylase Concentrations. Four in vitro experiments were conducted to assess the resiliency of SA activity in a spectrum of acid environments. High and low SA concentrations reflect the calculated maximum and minimum SA activities that were measured in premature infants. Exhaustive hydrolysis of the GP by excess SA concentration provided the standard for complete GP digestion in our system. An incubation of the GP without added SA activity controlled for possible acid cleavage.

High SA concentrations. To mimic the in vivo situation in which formula and saliva are mixed, we employed a modified soy milk formula and constructed a simulated saliva. The formula consisted of carbohydrate-free soy milk and $8 \mathrm{~g} / \mathrm{dl}$ of the ${ }^{14} \mathrm{C}$ GP substrate (specific radioactivity $=1.4 \mu \mathrm{Ci} / \mathrm{mg}$ ). The "saliva" was prepared by adding $0.5 \mathrm{mg}$ of human SA to $0.5 \mathrm{ml}$ of the $0.043 \mathrm{M} \mathrm{CaCl}_{2}$ solution; $0.05 \mathrm{ml}$ of this solution were then added to $1.2 \mathrm{ml}$ of Ringer's solution providing $1.1 \mathrm{U} \mathrm{SA} / \mathrm{ml}$ of the final reaction mixture. We estimated this level to be the maximum found in premature infants' saliva. To initiate digestion in the simulated oropharyngeal phase, $3.75 \mathrm{ml}$ of the formula were mixed with $1.25 \mathrm{ml}$ of the "saliva." The mixture was incubated at $37^{\circ} \mathrm{C}$ and constantly stirred. After $15 \mathrm{~s}$, a $0.1-\mathrm{ml}$ aliquot was removed for analysis. GP breakdown in this sample represents the accomplishments of SA within the oropharyngeal phase.

To initiate digestion in the simulated gastric phase, four $0.925-$ $\mathrm{ml}$ samples were removed from the remaining $4.9 \mathrm{ml}$ of salivaformula mixture and placed into individual test tubes containing $20 \mathrm{U}$ of pepsin in $0.75 \mathrm{ml}$ of dilute hydrochloric acid. The hydrochloric acid content was selected to provide pHs of 2, 3, 4, or 5 after the addition of the oropharyngeal phase aliquot; the $\mathrm{pH}$ stabilities of these solutions were confirmed periodically during the incubation. Separate experiments were utilized to evaluate the extent of hydrolysis during a gastric incubation lasting either $5 \mathrm{~min}$ or $3 \mathrm{~h}$.

After removal of a $0.1 \mathrm{ml}$ aliquot at 0 time, the intestinal phase of digestion was initiated by the addition of $0.175 \mathrm{ml}$ of a solution containing sufficient cholate, chymotrypsin, and trypsin to provide concentrations of $4.0 \mathrm{mM}$ of cholate and $25 \mathrm{U} / \mathrm{ml}$ of both enzymes. Varying amounts of sodium carbonate were included to return the $\mathrm{pH}$ to 7.0 . One hundred microliter samples were removed at $0,15,60$, and $180 \mathrm{~min}$ of incubation. Hydrolysis in the 0 time sample represents the extent of hydrolysis through the end of the gastric phase; hydrolysis in subsequent samples represents digestion in the intestinal phase.

Low SA concentrations. An experiment similar in design to that described above was performed with a SA concentration of $0.011 \mathrm{U}$ of $\mathrm{SA} / \mathrm{ml}$ of formula. Incubation times in this study were $15 \mathrm{~s}$ for the oropharyngeal phase, $3 \mathrm{~h}$ for the gastric phase, and $3 \mathrm{~h}$ for the intestinal phase. Gastric phase studies were run only at $\mathrm{pH} 5$.

Excess $S A$ concentrations (exhaustive hydrolysis). The oropharyngeal phase was prepared as in the high SA experiment. $\mathrm{HCl}$ was not added in the gastric phase. At the conclusion of a 3-h intestinal phase $55 \mathrm{U}$ (a 100 -fold excess) of SA was added to a $0.1 \mathrm{ml}$ sample of the reaction mixture and a further 20-min incubation was completed to ensure exhaustive SA action on the GP substrate.

No SA (acid hydrolysis). An incubation was performed to determine the extent of acid hydrolysis of the GP in our in vitro system when SA was withheld. The protocol described in the high SA experiment was followed without the addition of SA; seven studies using 3-h gastric and intestinal phases were performed. Gastric phase $\mathrm{pH}$ in the seven tests was varied: $\mathrm{pH} 2$ in three and pHs $3,4,5$, and 6 in one study each.

Quantification of the hydrolysis. All incubation periods were terminated by the addition of $10 \mu \mathrm{l}$ of a $20 \mathrm{~g} / \mathrm{dl}$ solution of trichloroacetic acid to $100 \mu \mathrm{l}$ of the reaction mixture. After centrifugation at $15,000 \mathrm{rpm}$ for $20 \mathrm{~min}, 20 \mu \mathrm{l}$ of the supernatant were applied to the LK-5 thin-layer plates; adjacent lanes contained $50 \mu \mathrm{g}$ of the partial corn starch hydrolysate as standards. The plates were developed with ethyl acetate:methanol:water $(74: 80: 46)$. After $150 \mathrm{~min}$ of development followed by thorough drying, the plates were scored with a diamond. The lanes containing the standards were separated and stained with the anisaldehyde reagent (8) to locate oligomers with DPs of 1-9; the area of silica gel containing these oligomers was scraped from the test lanes and collected into a vial for scintillation counting (Fig. 2). The remainder of the lane, i.e. the area between DP 9 and the origin, was then scraped into a second vial. After the addition of $1 \mathrm{ml}$ of water, the scintillation bottles were vigorously shaken and 10 mls of Scintiverse Bio-HP were added. Radioactivity was determined in a Beckman LS 75 Scintillation spectrometer. We have defined the extent of GP hydrolysis by the 
following relationship:

$$
\% \text { GP hydrolysis }=\frac{\mathrm{cpm} \text { in area of DPs } 1-9}{\mathrm{cpm} \text { in total lane }} \times 100
$$

Comparison of Adult and Premature SA. Saliva from two 1wk-old premature infants (gestational age $30 \mathrm{wk}, 1000 \mathrm{~g}$; gestational age $24 \mathrm{wk}, 612 \mathrm{~g}$ ) was collected and diluted in normal saline. After centrifugation at $15,000 \times g$, the supernatants were passed through a $0.45-\mu$ membrane filter and the SA activity $/ \mathrm{ml}$ saliva was determined with the Phadebas test.

Electrophoresis of amylase was performed according to the technique of Leclerc and Forest (9). Into individual wells of electrophoretic agar gel plates were placed the following samples: 0.01 and $0.02 \mathrm{U}$ of SA activity from each premature infant; 0.01 and $0.02 \mathrm{U}$ of standard adult SA; and $0.14 \mathrm{U}$ of porcine pancreatic amylase.

To assess the acid resistance and the hydrolytic capabilities of premature and adult SA, an in vitro comparison was made. Samples $(1.25 \mathrm{ml})$ of ${ }^{14} \mathrm{C} \mathrm{GP} /$ carbohydrate-free formula (specific activity $10.8 \mathrm{mCi} / \mathrm{g}$ ) were mixed in each of two test tubes with $0.33 \mathrm{U}$ of either premature SA or standard human SA (Sigma) and incubated under the conditions outlined in the high SA experiment above. The 3 -h gastric phase was conducted at $\mathrm{pH}$ 3.5; after the 3-h intestinal phase the resulting oligomeric profile was assessed by thin-layer chromatography and scintillation spectroscopy.

Analysis of Data. The correlation of gestational age with SA concentration in saliva was analyzed by multiple linear regression. The relationship between $\mathrm{pH}$, incubation times, and the extent of GP digestion was examined by two-way analysis of variance. Hydrolysis at low concentrations of SA was compared with that of the SA free control by the Student's $t$ test.

\section{RESULTS}

Comparison of Radioactive versus Nonradioactive Glucose Polymer (DP 18-29). Both the radioactive and nonradioactive $\mathrm{DP}_{\wedge v \mathrm{~V} ;} 23$ eluted from the Bio-Gel P-2 column between 6.4 and $7.9 \mathrm{ml}$, a result that indicates that they are similar in size. As shown in Figure 1, the amylase digestion products of the radioactive and nonradioactive substrate have an essentially identical elution profile from Bio-Gel P-2 columns.

SA Activity in Premature Saliva. The range of SA activity was 0.6 to $31 \mathrm{U} / \mathrm{ml}$ saliva. In general, higher SA values were seen with advanced gestational age, but linear regression analysis did not show a significant correlation $\left(r^{2}=0.553\right)$.

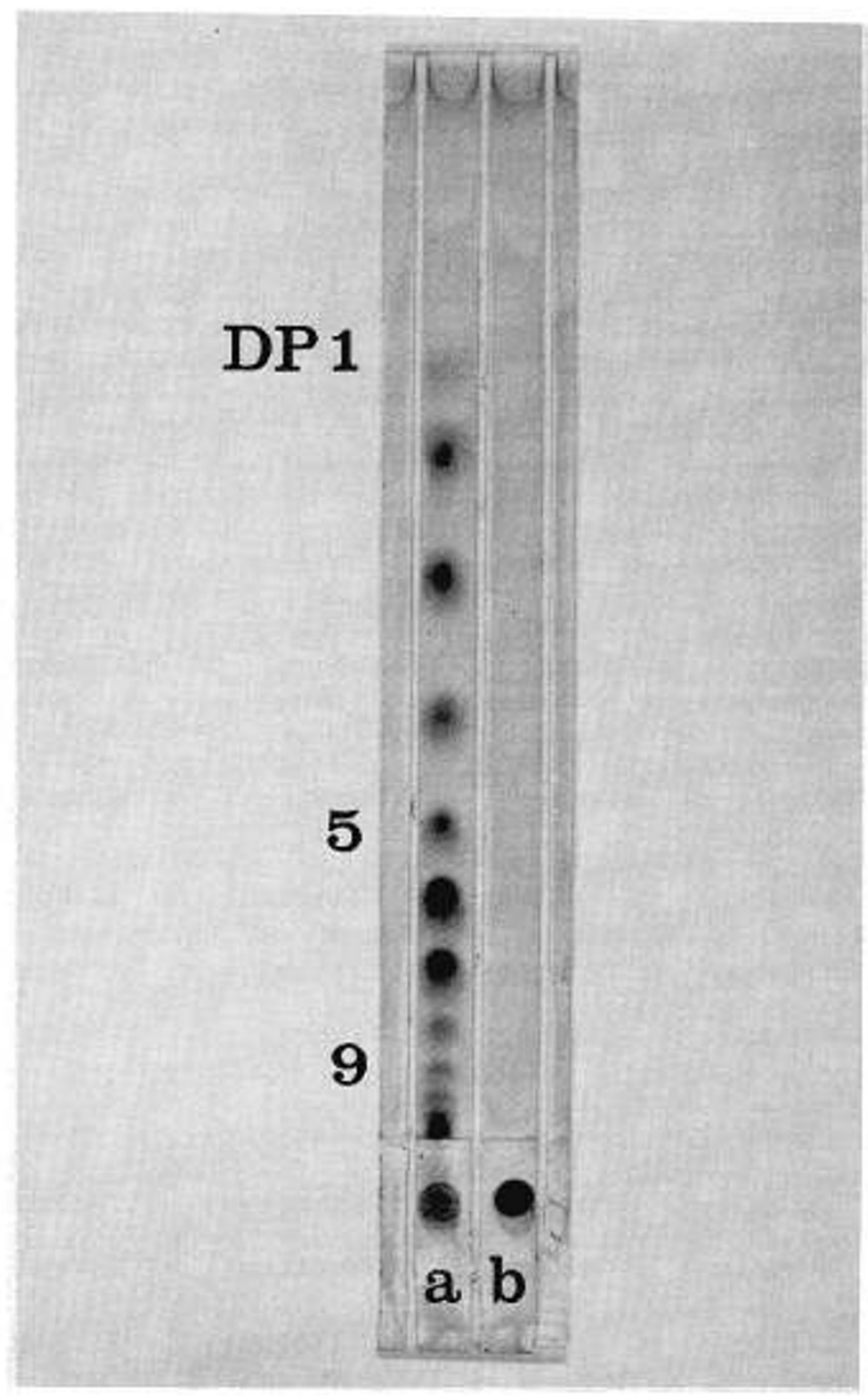

Fig. 2. Typical thin-layer chromatographic separation of glucose oligomers and polymers. Lane $A$, partial corn starch hydrolysate; lane $B$, $\mathrm{DP}_{A V G} 23$. Percent breakdown of $\mathrm{DP}_{\mathrm{AVG}} 23$ is defined as the ratio of $\mathrm{cpm}$ in DP's 1-9 to total cpm in the lane.

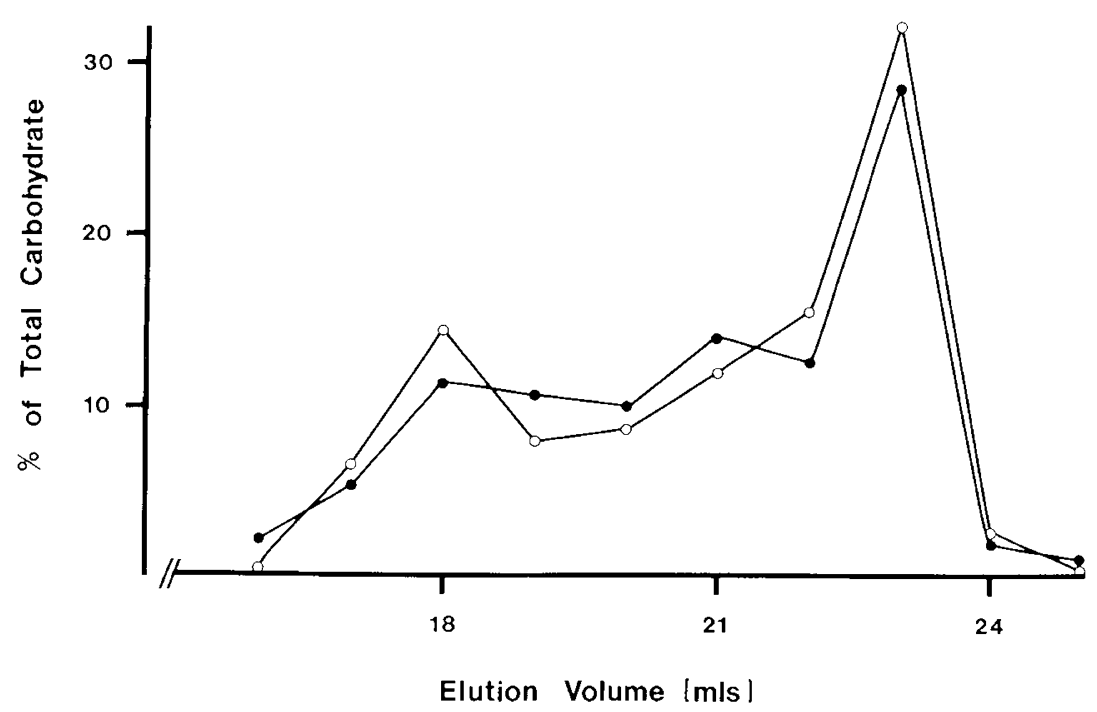

Fig. 1. Elution profile of a mixture of nonradioactive and ${ }^{14} \mathrm{C}$-labeled $\mathrm{DP}_{\mathrm{Avg}} 23$ from a $38.5 \times 0.7 \mathrm{~cm}$ Bio-Gel P-2 column. The open circles represent the profile of radioactivity while the closed circles represent the profile of carbohydrate. 
$G P$ Hydrolysis Experiments. High $S A$ concentration. In the 15 -s oropharyngeal phase less than $1 \%$ digestion of GP occurred. The 0.5 -min sample of the intestinal phase, which represents the extent of gastric phase digestion, is illustrated in Figures 2 and 3 and in Table 1. Following a brief gastric exposure $(5 \mathrm{~min})$ at acid conditions, the percent of GP hydrolyzed is only $2.1 \%$, even at $\mathrm{pH} 5$. When the gastric incubation time was extended to $3 \mathrm{~h}$, there was no significant hydrolysis at $\mathrm{pH} 2$ or 3; however, at $\mathrm{pH}$ 4 or 5 digestion proceeded and 5.1 and $13.5 \%$, respectively, of the total GP were digested. The prior gastric phase $\mathrm{pH}$ strongly influenced the rate and extent of GP digestion during the intestinal phase. The effect was evident even with a gastric phase exposure of only 5 min (Figs. 3 and 4). A 5 -min gastric phase exposure at $\mathrm{pH} 2$ and 5 resulted in intestinal phase digestions of 3.9 and $12.2 \%$, respectively, after $180 \mathrm{~min}$; similarly, after a gastric phase of $3 \mathrm{~h}$ at the same $\mathrm{pHs}$, the percentage breakdown after $180 \mathrm{~min}$ of the intestinal phase was 7.1 and $23.8 \%$, respectively. Two-way analysis of variance examining the effects of gastric phase $\mathrm{pH}$ and gastric incubation time on GP digestion confirms the significance of both factors $(p<0.001)$.

Low SA concentration. At the lowest level of SA studied none of the GP was hydrolyzed during a 3-h gastric phase at $\mathrm{pH} 5$. However, in the subsequent $3 \mathrm{~h}$ of the intestinal phase up to $5 \%$ of the GP was digested (mean $\pm \mathrm{SD}, 3.96 \pm 0.75 \%$ ). Although this degree of hydrolysis is limited, by comparison with the SAfree control, it represents significant hydrolysis $(p<0.001)$.

Exhaustive hydrolysis of the GP. In samples maintained at $\mathrm{pH}$ 6.4 for $360 \mathrm{~min}$, the time equal to the combined gastric and

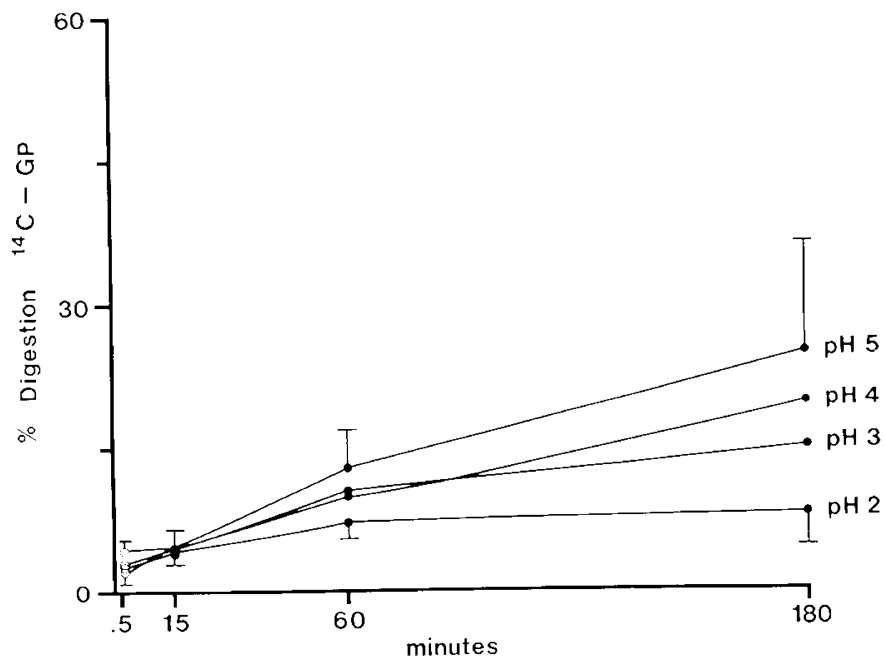

Fig. 3. Time course of the digestion of the glucose polymer $\mathrm{DP}_{\mathrm{AvG}} 23$ in the intestinal phase following $5 \mathrm{~min}$ of exposure in the gastric phase at the four $\mathrm{pHs}$ shown. The open circles represent hydrolysis within the gastric phase while the closed circles represent subsequent intestinal phase cleavage. intestinal phases, $29 \%$ of the GP was digested. The addition of a 100 -fold increment of SA $(11 \mathrm{U} / 100 \mu \mathrm{l})$ increased the percent digestion to $42 \%$. This value was taken to represent the maximum possible endoamylase digestion (to DPs 1-9) in this system and was used to normalize the data in all hydrolysis studies.

GP hydrolysis by acid. In the absence of SA, the extent of GP hydrolysis ranged between 1.8 and $2.3 \%$ for gastric phase pHs ranging from $2-6.4$, i.e. there is no significant breakdown even at the low $\mathrm{pH}$.

Adult versus premature $S A$. The SA activity in the saliva of the two premature infants was 1 and $2 \mathrm{U} / \mathrm{ml}$, respectively. The electrophoretic isoenzyme pattern of the premature and adult SA was identical. All samples contained three isoenzymes which migrated equivalent distances toward the anode. As shown in

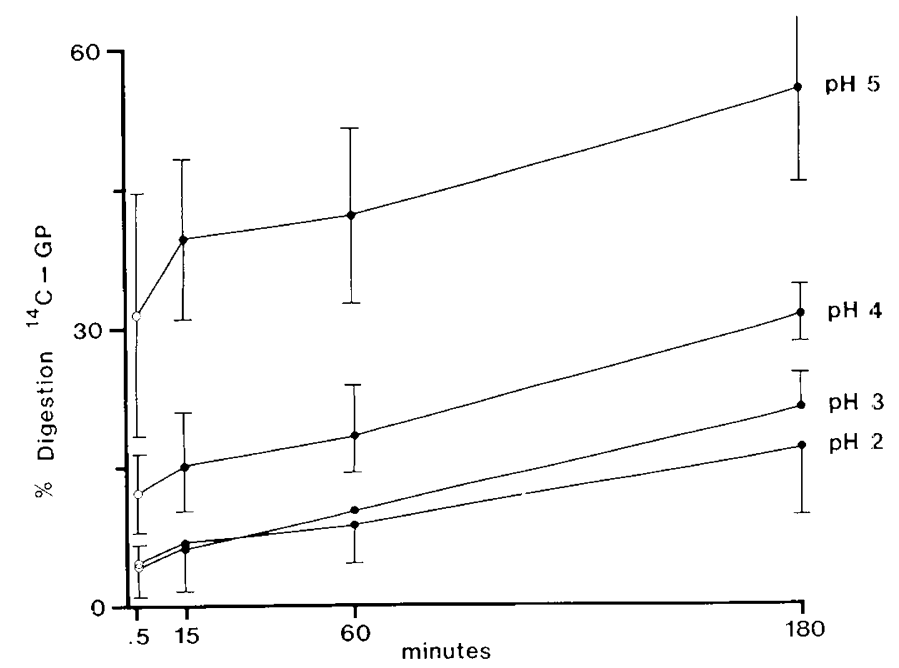

Fig. 4. Time course of the digestion of the glucose polymer $\mathrm{DP}_{\mathrm{AvG}} 23$ in the intestinal phase following $180 \mathrm{~min}$ of exposure in the gastric phase at the four $\mathrm{pHs}$ shown. The open circles represent hydrolysis within the gastric phase while the closed circles represent subsequent intestinal phase coverage.

Table 2. Comparison of the distribution of oligosaccharides produced by the action of premature or adult salivary amylase on the long-chain glucose polymer, $D P_{\mathrm{AvG}} 23$

\begin{tabular}{ccc}
\hline & \multicolumn{2}{c}{ Salivary amylase } \\
\cline { 2 - 3 } Digestion products & Premature & Adult \\
\hline DP 1-4 & $36.0^{*}$ & 35.0 \\
DP 5-8 & 5.4 & 6.8 \\
DP 9-10 & 3.5 & 3.6 \\
DP 11-16 & 10.6 & 11.4 \\
DP > 16 & 44.5 & 43.0 \\
\hline
\end{tabular}

* Results are expressed as a percent of the total.

Table 1. Effect of the pII and duration of the gastric phase on the extent of hydrolysis of the glucose polymer DP $P_{\wedge \mathrm{vG}} 23$ by salivary amylase during the subsequent intestinal phase

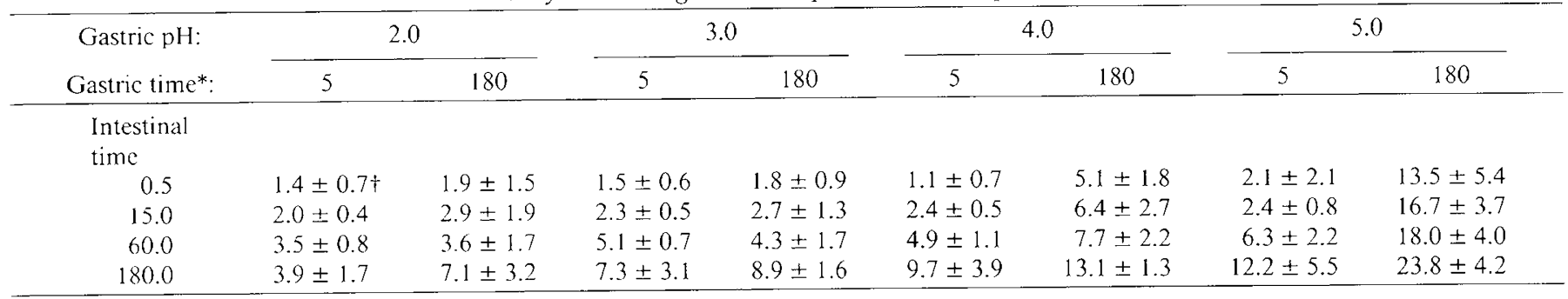

* Times are expressed in min.

$\uparrow$ Percent of glucose polymer hydrolyzed \pm SEM. 
Table 2, the distribution of oligosaccharides produced by the action of premature or adult $\mathrm{SA}$ on $\mathrm{DP}_{\mathrm{AvG}} 23$ was quite similar.

\section{DISCUSSION}

In this study we sought to quantify the potential contribution of salivary amylase activity toward GP assimilation in prematures. Pancreatic amylase, the enzyme normally responsible for hydrolysis of GPs, is absent in the neonatal period and its activity is only detected after 4 to 6 months of life. Because infants appear to assimilate GPs, the absence of the most effective endoamylase raises questions concerning the alternative mechanisms by which these polymers are cleaved early in life. Since Skude and Ihse (10) first demonstrated, in adults, that $15 \%$ of the endoamylase activity present in duodenal aspirates is of salivary origin, the tenet that gastric acidity is an absolute barrier to salivary amylase survival has been in question. This persistence of salivary amylase activity in the small intestine suggests that it could contribute to intestinal GP digestion. Although the relative amount of salivary amylase activity in the duodenum of persons with normal pancreatic amylase levels is quantitatively low (mean $=90 \mathrm{U} / \mathrm{ml}$ saliva in adults), the role of salivary amylase in the initial 6 months of life might be substantial, especially with the "benign" gastric milieu found in neonates (11). Hypothesizing that salivary amylase survives in the stomach of prematures, Hodge et al. (12) sampled gastric contents following feedings. The interfeeding $\mathrm{pH}$ remained above 3 for over $2 \mathrm{~h}$ in most instances and often never fell below that level. Amylase activity was measurable in the gastric contents provided the $\mathrm{pH}$ remained greater than 3.0. Nonetheless, Hodge et al. (13) did not demonstrate digestion products in stomachs of newborns who were orally fed corn syrup solids.

Premature saliva contains very low levels of salivary amylase activity $(0.6-33 \mathrm{U} / \mathrm{ml})$, and its effectiveness under the varying conditions in the oropharynx, stomach, and small bowel has not been measured. Having manufactured a uniformly labeled ${ }^{14} \mathrm{C}$ GP, we developed a sensitive assay to quantify the products of salivary amylase activity as digestion progresses through simulated oropharyngeal, gastric, and intestinal environments. Our results suggest the extent to which salivary amylase, at concentrations found in premature infants, can act as a substitute for pancreatic amylase in the digestion of GPs. This study establishes several points: 1) over $40 \%$ of the products of exhaustive digestion of the GP by excess salivary amylase are between 1 and 9 glucose units in length. Nearly $60 \%$ remain as products greater than DP10 in length. This result represents the maximum possible digestion by salivary amylase activity under our in vitro conditions and provides the standard against which we compared digestion in the various simulated intestinal phases. 2) Slightly more than half of the potentially digestible long-chain glucose polymers are hydrolyzed when the salivary amylase concentration is maximal, the gastric $\mathrm{pH}$ is high, and the incubation time is prolonged. 3) In studies employing the high salivary amylase concentrations, GP digestion (in both the gastric and subsequent intestinal phase) is a function of both the gastric $\mathrm{pH}$ and incubation time. 4) In studies in which the minimum salivary amylase concentration was used, digestion was insignificant regardless of $\mathrm{pH}$ or duration of incubation. 5) The GP is resistant to mild acid hydrolysis, even at $\mathrm{pH} 2$.

While this study suggests that the contribution of salivary amylase activity to GP digestion is limited under the in vitro conditions used, it may underestimate the enzyme's contribution to GP assimilation in vivo for a number of reasons: milk curd formation in the stomach may foster hydrolysis by offering a protected niche with low acidity; in the small bowel, the products of endoamylase activity are continually removed due to brush border glucoamylase activity and glucose absorption, processes that prevent product inhibition; additionally, the salivary amylase concentrations used in our study may not be representative of those during actual feeding situations. Saliva output and amylase concentrations vary with the type of stimulus (14) and may be higher during actual feedings. Conversely, the longer incubation times used in this study probably overestimate the anticipated small bowel transit time. Gastric contents normally empty within 100 min after feeding, and despite a dearth of accurate information on small bowel transit times, a 3-h intestinal phase is probably somewhat long (15).

In order to ensure a sufficient quantity of uniform salivary amylase for our studies, we used commercial, adult, human salivary amylase. We established that salivary amylase from premature infants, when compared to the adult salivary amylase, had an identical electrophoretic isozymic pattern. Under our in vitro conditions of digestion, premature and adult SA had identical acid resistance and produced a similar pattern of oligomer digestion products from $\mathrm{DP}_{\mathrm{AvG}} 23$.

In our study we have defined endoamylase activity as the production of oligomers with DPs' ranging from 1 through 9. More recently, however, using a modified thin-layer system, we have shown that after exhaustive hydrolysis as much as $30 \%$ of the long-chain polymer $\left(\mathrm{DP}_{\mathrm{AVG}} 23\right)$ is broken down to oligomers with DPs between 10 and 18 . These molecules may be limit dextrins whose further digestion would normally be promoted by intestinal isomaltase.

The results of this investigation contribute to an emerging picture of GP assimilation in newborns. Four enzymes, or enzyme systems, have been cited as possible substitutes for pancreatic amylase: salivary amylase, breast milk amylase, the enzymes of the mucosal brush border, and the colonic bacterial enzymes. This study focused on the accomplishments of salivary amylase and suggests that under optimal conditions the potential contribution of its activity to GP digestion are significant. In newborn premature infants, however, digestion accomplished by salivary amylase falls far short of what pancreatic amylase presumably achieves in later life. In contrast to available salivary amylase activity in the newborn, the amylase activity of breast milk is substantial, but breast-fed infants rarely receive GPs. To evaluate whether brush border glucoamylase activity can fully digest long-chain GPs, we have previously employed canine jejunal Thiry-Vella fistulae free of endoamylase activity. In this preparation, which depends on glucoamylase activity for GP assimilation, we demonstrated that the brush border oligosaccharidases have a limited ability to digest long-chain GPs, a finding in agreement with Alpers' suggestion that glucoamylase activity is best suited for the hydrolysis of oligomers between DPs 5 and 9 (16). The relevant enzymes available for polymer digestion in the newborn period must act in concert. In addition there is little doubt that the colonic flora, which are important for lactose absorption by the premature infant (17), also play a role in GP assimilation (18). In the newborn, who assimilates GP without manifesting diarrhea, one can assume that when salivary amylase activity is low, the colonic flora convert the carbohydrate to readily absorbed short-chain fatty acids.

Acknowledgments. The authors thank Constance Seckel for technical assistance; Lisa Evers, Brenda Gaffney, and Rita Compston for preparation of the manuscript; John Hayes for performing the statistical analyses; and Sonia Kerzner for preparation of the illustrations.

\section{REFERENCES}

1. Lee PC 1982 Alternate pathways in starch digestion - their importance in premature and young infants. In: Lifshitz $\mathrm{F}$ (ed) Carbohydrate Intolerance in Infancy. Marcel Dekker Inc, New York, pp 223-233

2. Grand RJ, Watkins JB, Torti FM 1976 Development of the human gastrointestinal tract. Gastroenterology 70:790-810

3. Porath J 1963 Some recently developed fractional procedures and their application to peptide and protein hormones. Pure Appl Chem 6:233--244

4. Sloan HR, Kerzner B, Seckel C 1984 Large scale preparation of selected glucose oligomers and polymers by gel filtration chromatography. Prep Biochem $14: 245-256$ 
5. Collares EF Brasiz M do RL, Kawasaki eST 1979 Secrecao de saliva, concentracao e secrecao da amilase salivar humana no primeiro ano de vida. Arq Gastroenterol 16:91-94

6. Collares EF, Brasil M doRL, Fernandes MIM 1980 Secrecao de saliva, concentracao e secrecao da amilase salivar em recem-nascidos de baix o pesa. Arq Gastroenterol 17:224-227

7. Gryboski JD 1969 Suck and swallow in the premature infant. Pediatrics 43:96102

8. Kwiterovich PO Jr. Sloan HR, Fredrickson DS 1970 Glycolipids and other lipid constituents of normal human liver. J Lipid Res 11:322-330

9. Leclerc P. Forest JC 1982 Electrophoretic determination of isoamylases in serum with commercially available reagents. Clin Chem 28:37-40

10. Skude G, Ihse I 1976 Salivary amylase in duodenal aspirates. Scand J Gastroenterol 11:17-20

11. Lebenthal E Lee PC, Heitlinger LA 1983 Impact of development of the gastrointestinal tract on infant feeding. J Pediatr 102:1-9

12. Hodge C, Lebenthal E, Lee PC, Topper W 1983 Amylase in the saliva and in the gastric aspirates of premature infants: its potential role in glucose polymer hydrolysis. Pediatr Res 17:998-1001

13. Hodge C, Lee PC, Topper W, Lebenthal E 1982 Digestion of corn syrup sugars in neonates: importance of salivary amylase and gastric hydrolysis. Pediatr Res 16:166A(abstr)

14. Afonsky D 1961 Secretion of saliva. In: Afonsky D (ed) Saliva and Its Relation to Oral Health: A Survey of the Literature. University of Alabama Press. Mobile, pp 1-21

15. Siegel M, Lebenthal E, Krantz B 1984 Effect of caloric density on gastric emptying in premature infants. J Pediatr 104:118-122

16. Kerzner B, Sloan HR, Haase GL, McClung HJ, Ailabouni A 1981 The jcjunal absorption of glucose oligomers in the absence of pancreatic enzymes. Pediatr Res 15:250-253

17. MacLean WC Jr, Fink BB 1980 Lactose malabsorption by premature infants: magnitude and clinical significance. J Pediatr 97:383-385

18. Shulman RJ, Wong WW, Irving CS, Nichols BL, Klein PD 1983 Utilization of dietary cereal by young infants. J Pediatr 103:23-28 